\title{
Percepções de coordenadores de unidades de saúde sobre a fitoterapia e outras práticas integrativas e complementares
}

\author{
Perceptions of health unit coordinators on Phytotherapy and other integrative \\ complementary practices
}

Dayane Cordeiro Machado', Silvia Beatriz Costa Czermainski², Edyane Cardoso Lopes ${ }^{3}$

'Especialista em Saúde da Família e Comunidade pelo Grupo Hospitala

Conceição (GHC) - Porto Alegre (RS), Brasil.

Farmacêutica da Secretaria Especial de Saúde

Indígena do Ministério da Saúde - Cascavel

(PR), Brasil.

dayane.machado@saude.gov.br

${ }^{2}$ Mestre em Gestão da Assistência Farmacêutica pela Universidade Federal do Rio Grande do Sul (UFRGS) - Porto

Alegre (RS), Brasil. Coordenadora do Núcleo de Estudos e Pesquisas em Assistência Farmacêutica da Escola de Saúde Pública do Rio Grande do Sul.

silvia-czermainski@saudersgovbr

${ }^{3}$ Doutoranda em Epidemiologia pela Universidade Federal do Rio Grande do Sul (UFRGS) - Porto Alegre (RS), Brasil. Farmacêutica da Secretaria Estadual de Saúde do Rio Grande do Sul, Coordenação da Política de Assistência Farmacêutica - Porto Alegre (RS) - Brasil.

edyane-lopes@saude.rs.gov.br

\begin{abstract}
RESUMO As práticas integrativas e complementares estão em fase de expansão. O estudo objetivou conhecer o ambiente entre gestores para a inclusão de fitoterápicos na assistência. Nesta série de casos, descrevemos as percepções de coordenadores de unidades de saúde sobre as práticas integrativas. Os dados foram coletados em um questionário estruturado. O interesse pela inserção das terapias foi demonstrado por 13 dos 15 entrevistados, e o mesmo número relatou uso de fitoterapia. A fitoterapia também é indicada aos usuários por 13 dos coordenadores. O grupo percebe haver maior prescrição de plantas medicinais do que de fitoterápicos. Conclui-se que há boa perspectiva de aceitação da fitoterapia na rede.
\end{abstract}

PALAVRAS CHAVE: Fitoterapia; Terapias complementares; Atenção primária.

ABSTRACT The complementary and integrative practices are undergoing expansion. This study aimed to investigate the environmental among managers for the inclusion of herbal medicines. In this case series we describe the perceptions of coordinators of health services on integrative practices. Data were collected on a structured questionnaire. Interest in the integration of alternative therapies was demonstrated by 13 of the 15 respondents and the same number reported use of herbal medicine. Herbal medicine is indicated to users by 13 coordinators. The group realizes that there is a higher prescription of medicinal plants than herbal. It is concluded that there is good acceptance of it in the network.

KEYWORDS: Phytoterapy; Complementary therapies; Primary Health Care. 


\section{Introdução}

A demanda pela implantação da Fitoterapia no Sistema Único de Saúde (SUS) resultou em algumas políticas públicas nacionais e estaduais, e em várias iniciativas municipais no Brasil. A Política Nacional de Plantas Medicinais e Fitoterápicos (PNPMF) e a Política Nacional de Práticas Integrativas e Complementares (PNPIC) no SUS encontram-se em fase de implementação e têm suscitado reflexôes sobre as dificuldades encontradas quanto ao conhecimento, entendimento e à aceitação dessas práticas. Nos países em que a principal forma de medicina praticada é a alopática, outras formas de atenção à saúde são consideradas complementares ou alternativas (KUREBAYASHI et al, 2008). Para Souza e Luz (2009), as novas práticas terapêuticas suprem uma demanda social, pois oferecem outras formas de solução ou alívio para a questão do sofrimento.

Tesser e Barros (2008), com relação às classificaçôes de práticas empregadas na medicina alternativa e complementar, especificam que;

\section{[...] quando essas práticas são usadas juntas} com práticas da biomedicina, são chamadas complementares; quando são usadas no lugar de uma prática biomédica, consideradas alternativas; e quando são usadas conjuntamente baseadas em avaliaçóes cientificas de segurança e eficácia de boa qualidade, chamadas integrativas. (TESSER; BARROS, 2008, p. 916).

O governo brasileiro, a exemplo da Organização Mundial da Saúde (OMS), tem elaborado uma série de documentos que reforçam o desenvolvimento de políticas públicas a fim de inserir no sistema oficial de saúde dos seus Estados-membros práticas de medicina complementar e integrativa (BRASIL, 2006a; BRASIL, 2006b; BRASIL, 2006c; ORGANIZAÇÃO MUNDIAL DE SAÚDE; FUNDO DAS NAÇÔES UNIDAS PARA INFÂNCIA, 1979; ORGANIZACIÓN MUNDIAL DE LA SALUD, 1991).

No Brasil, a intenção de ampliar as opçóes terapêuticas do SUS é marcada pela construção da PNPIC, na qual está incluída a fitoterapia (BRASIL, 2006b). A fitoterapia caracteriza-se pelo uso de plantas medicinais em suas diferentes formas de apresentação, sem a utilização de substâncias ativas isoladas, mesmo que sejam de origem vegetal (LUZ NETTO, 1998). No Brasil, o uso de plantas medicinais é influenciado pelo conhecimento acumulado das culturas indígenas, europeias e africanas (SANTOS, 2000; SANTOS, 2008).

Considerando as recomendaçóes da OMS, a cultura e a biodiversidade do Brasil, assim como a complexidade que envolve a fitoterapia, a PNPMF objetivou a ampliação do acesso a plantas medicinais, fitoterápicos e serviços relacionados à fitoterapia voltada para a segurança, eficácia, qualidade e integralidade da atenção à saúde dos brasileiros, além do desenvolvimento da cadeia produtiva e da indústria nacional (BRASIL, 2006a). Czermainski (2010, p. 137) analisa o processo de formulação da PNPMF e a identifica como um avanço do movimento popular. A autora reforça a importância do diálogo entre os atores envolvidos na construção da referida política intersetorial para o aperfeiçoamento de suas diretrizes e efetividade das açôes.

As instâncias de controle social reivindicaram e deliberaram sobre a inclusão da fitoterapia no SUS, como observado nos relatórios das $8^{\mathrm{a}}$ e $10^{\mathrm{a}}$ Conferências Nacionais de Saúde (BRASIL, 1986; BRASIL, 1996), e foi amplamente discutida no processo da I Conferência Nacional de Medicamentos e Assistência Farmacêutica - CNMAF (BRASIL, 2005). Entre as propostas aprovadas na I CNMAF, destacam-se aqui:

Fazer gestóes junto aos órgáos competentes para adotarem medicamentos fitoterápicos no elenco da Assistência Farmacêutica Básica (AFB), estimular o uso racional destes medicamentos, por meio de uma política de informaçóes e garantir o acesso aos usuários do SUS.

Definir e normatizar os serviços de fitoterapia, organizados por nivel de complexidade da atenção à saúde, com recursos humanos qualificados, incorporando os conhecimentos tradicionais. (BRASIL, 2005).

O Conselho Nacional de Saúde, ao aprovar a Política Nacional de Assistência Farmacêutica, em 2004, 
recomendou a incorporação dessa opçáo terapêutica basead a no incentivo à produção nacional, com a utilização da biodiversidade existente no país (BRASIL, 2004b). A proposta de inclusão de fitoterápicos na assistência farmacêutica pública ganha sustentação a partir de 2007, com a seleção de dois produtos do elenco de referência nacional de medicamentos (BRASIL, 2007). Esse número foi ampliado para oito plantas medicinais em apresentaçóes diversas, que podem receber financiamento pelas normas do Componente Básico da Assistência Farmacêutica. São elas: espinheira-santa, guaco, alcachofra, aroeira, cáscara-sagrada, garra-dodiabo, isoflavona-de-soja e unha-de-gato (BRASIL, 2010c). A inclusão de fitoterápicos na assistência farmacêutica básica supre algumas das indicaçôes que carecem de opçóes nas listas de medicamentos básicos de alguns municípios.

Muitas secretarias municipais e estaduais já apresentavam programas de fitoterapia com uso de recursos próprios, anteriormente à inclusão dessa terapêutica no âmbito nacional (PIRES; BORELLA; RAYA, 2004; GRAÇA, 2004; OGAVA et al, 2003; REIS et al, 2004; MICHILES, 2004; CARNEIRO et al, 2004; RIO DE JANEIRO, 1996; RIO DE JANEIRO, 2001).

É importante destacar a interação de programas de fitoterapia com outros programas intersetoriais, em especial, com a cadeia produtiva, como observado nas experiências dos estados do Rio de Janeiro e do Mato Grosso (REIS et al, 2004; MATO GROSSO, 2005). Programas vinculados a universidades, como o programa 'Farmácias Vivas', são estímulos para o uso correto de plantas medicinais selecionadas por sua eficácia e segurança em substituição ao rotineiro uso empírico de plantas pelas comunidades (MATOS, 1998; SILVA; GONDIM; NUNES, 2006).

$\mathrm{O}$ crescente interesse pela inserção da fitoterapia no SUS pode ter várias justificativas além da economia de custos de aquisição de produtos. Apresenta-se como uma alternativa para geração de emprego, melhora da qualidade de fitoterápicos acessados pela população, menor incidência de efeitos colaterais; são alternativas consideradas mais 'suaves' de tratamento quando comparadas aos medicamentos convencionais (REIS et al, 2004; PIRES; BORELLA; RAYA, 2004; OGAVA et al,
2003). Para Silva, Gondim e Nunes (2006), os fitoterápicos serviram para suprir a lacuna deixada pela escassez de medicamentos alopáticos nas unidades de saúde.

Czermainski (2010) destaca a importância da definição de qual fitoterapia é proposta para o SUS e percebe que as propostas de inserção advindas das organizaçóes populares são diferentes das originárias de organizaçóes farmacêuticas, por exemplo. Fonte et al (2005), em debate sobre a complexidade em torno do uso de plantas medicinais e fitoterápicos, observam diferentes olhares, entendimentos, interesses e concepçôes.

Apesar do reconhecimento da fitoterapia por conselhos profissionais da saúde, como os da medicina, enfermagem e farmácia, muitos profissionais se sentem inseguros para abordar esse assunto (CFM, 1992; CFF, 2007a; COFEN, 1997; ALVIM et al, 2006; PONTES; MONTEIRO; RODRIGUES, 2006). Nesse sentido, Souza e Vieira, citados por Rosa et al (2007), ressaltam a importância de espaços de discussáo sobre o tema, tanto no âmbito acadêmico quanto no de serviços, além da promulgação legal para garantir a sua oferta.

Michiles (2004) identifica a necessidade de um maior comprometimento dos gestores para o cumprimento de orientações oficiais sobre o tema fitoterapia. A autora afirma, também, que a maioria dos gestores desconsidera que ações de apoio a programas de fitoterapia podem contribuir para o desenvolvimento tecnológico e para a independência econômica do país na área de medicamentos.

Continuando a reflexão sobre o papel dos gestores e a temática, destaca-se o papel dos gestores da 'ponta', os coordenadores de unidades de saúde. Ramires, Lourenção e Santos (2004) comentam sobre a potencialidade desses gestores em operar mudanças nos espaços de produção. Nesse sentido justifica-se o presente trabalho, o qual propóe conhecer se há um ambiente favorável entre gestores de unidades de saúde de atenção básica para a inclusão de fitoterápicos na assistência farmacêutica municipal e, assim, subsidiar açóes de planejamento local.

Este trabalho vem ao encontro das necessidades de investigaçôes apontadas por outros autores: analisar a perspectiva dos profissionais de saúde sobre o uso de MAC, a possibilidade de introdução dessas práticas 
nos serviços convencionais de saúde e a posição dos gestores e produtores de políticas públicas de saúde sobre a sua incorporação no Sistema Único de Saúde (SPADACIO; BARROS, 2008).

\section{Metodologia}

Trata-se de uma série de casos em que foi empregada a metodologia quantitativa, com dados obtidos através de questionários estruturados aplicados aos coordenadores de unidades de saúde em uma das oito Gerências Distritais de Saúde da Secretaria Municipal de Saúde de Porto Alegre (SMS/POA). O projeto foi aprovado pelo Comitê de Ética em Pesquisa da SMS/ POA, de acordo com a Resolução CNS no 196/06 e com emprego do termo de consentimento livre e esclarecido (TCLE).

A população estudada na pesquisa se constituiu dos 21 coordenadores das unidades de saúde dos dois modelos de atenção (ESF e UBS), e que trabalham em dois distritos sanitários da cidade. $\mathrm{O}$ território a que pertencem as unidades estudadas é constituído do maior aglomerado de vilas populares e loteamentos irregulares do município. A população da região é de 178.713 habitantes (IBGE, 2000), atendidos em nível de atenção primária por nove unidades da Estratégia de Saúde da Família (ESF), doze Unidades Básicas de Saúde (UBS) e equipes de apoio. O Distrito escolhido é território de formação da Residência multiprofissional pertencente à Secretaria Estadual de Saúde do Rio Grande do Sul, às quais as pesquisadoras têm acesso.

Os dados foram coletados a partir de um questionário elaborado pela autora e aplicado aos coordenadores presentes na reunião de Coordenação da respectiva Gerência Distrital (outubro de 2010), à qual compareceram 15 coordenadores. Foram coletadas informaçôes sobre o perfil dos coordenadores, seus entendimentos e percepçôes sobre a fitoterapia e outras terapias integrativas e complementares. Os dados foram analisados quantitativamente com relaçáo à distribuição de frequências utilizando-se o programa Microsoft Excel'. Na compilação dos dados sobre o conhecimento da PNPIC, as terapias citadas foram categorizadas com relação à inserção nessa Política.

\section{Resultados e discussão}

Participaram da pesquisa 15 do total de 21 coordenadores, sendo 8 coordenadores oriundos de ESF e 7 de UBS. Dos quinze participantes, 14 são do sexo feminino, correspondendo a 9 profissionais da área da enfermagem, 4 da medicina, 1 da nutrição e 1 de outra área de graduação náo especificada. A caracterização da maioria de coordenadores de unidades de saúde pertencente ao sexo feminino e graduada no curso de Enfermagem fora identificada também por outros pesquisadores, como Alves et al (2004), que observaram a tendência de gênero feminino na força de trabalho em saúde.

No que se refere à faixa etária dos coordenadores, identificaram-se 7 com idade entre 21 e 40 anos, 6 entre 41 e 60 anos e somente 2 pessoas com mais de 60 anos.

Entre os participantes, 8 encontram-se graduados há mais de 15 anos, 1 entre 10 e 15 anos, 3 entre 5 e 10 anos e 3 pessoas entre 1 e 5 anos. $O$ tempo no cargo de chefia é variável: 5 com menos de um ano, 3 entre 1 a 5 anos, 5 entre 5 e 10 anos e somente 2 pessoas com mais de 10 anos.

\section{Educação continuada e autoavaliação de conhecimentos}

A maioria dos coordenadores $(n=14)$ possui curso de pós-graduação em nível de especialização, o que demonstra o interesse pela continuidade da formação. Com relação à participação em atividades de capacitação sobre fitoterapia, o número foi bem reduzido, pois apenas uma pessoa afirmou ter participado de alguma atividade de educação sobre o tema. Quando questionados se possuíam informaçóes sobre a PNPIC, seis pessoas declararam positivamente. Como visto, apesar $\mathrm{da}$ PNPIC existir há mais de seis anos, poucos profissionais de saúde têm algum conhecimento sobre ela, a 
inferir-se pela presente pesquisa, o que justifica a necessidade de divulgação e de maior número de capacitaçóes relacionadas.

Os entrevistados foram questionados se possuíam interesse na incorporação de terapias complementares ou integrativas à rede de Atenção Primária à Saúde (APS) do seu município: 14 assinalaram interesse e uma pessoa não se manifestou. $\mathrm{Na}$ questão aberta sobre quais terapias recomendariam para a rede, as terapias citadas foram categorizadas em contempladas e não contempladas na PNPIC. Entre as terapias reconhecidas e contempladas na política, a fitoterapia foi citada por 10 , acupuntura por 5 e homeopatia por 4 . Achado semelhante a um trabalho investigativo sobre a aceitação de práticas não convencionais por estudantes de medicina, o qual revelou que mais de $50 \%$ dos futuros médicos indicariam ou apoiariam o uso da acupuntura e da fitoterapia por seus pacientes (KULKAMP, et al., 2007).

As demais terapias alternativas citadas, hidroginástica, equiterapia, musicoterapia e reiki, não constam naquela Política e foram relatadas por apenas um entrevistado. Esse conjunto de práticas necessita ser diferenciado entre racionalidades e técnicas terapêuticas, pois significa a incorporação de elementos de outra racionalidade médica.

O nível de conhecimento sobre terapias complementares e integrativas foi considerado básico por 9 dos coordenadores, e 4 consideraram seu conhecimento nulo; 1 como sendo médio e 1 avançado, o que reafirma a necessidade de capacitaçóes dos profissionais sobre o tema.

Quando questionados sobre a existência de terapias complementares e integrativas já disponíveis na rede de APS de Porto Alegre, 4 pessoas citaram a disponibilização da acupuntura e 2 da homeopatia. A referência para o atendimento em homeopatia no município é o Centro de Saúde Modelo, com atendimento médico e dispensação em farmácia homeopática. Esse Centro de Saúde é pioneiro no estado do RS na implantação oficial da homeopatia em uma unidade do SUS, de acordo com Santana, Hennington e Junges (2008). E o mesmo local disponibiliza acupuntura aos usuários (NEVES; SELLI; JUNGES, 2010).
Os coordenadores foram questionados se concordariam com a inserção de fitoterápicos na relação de medicamentos essenciais do município, sobre o que catorze dos quinze se manifestaram positivamente e apenas uma pessoa não declarou a sua opiniấo. De forma geral, a grande maioria dos coordenadores (n = 13) acredita que a fitoterapia pode complementar o tratamento do usuário, e para 6 dos entrevistados a fitoterapia não substitui os tratamentos convencionais. A fitoterapia foi apontada por 3 coordenadores como adequada para tratamento de doenças 'leves'.

A interação dos fitoterápicos com outros medicamentos não é considerada importante por 6 profissionais, enquanto 3 acreditam que a fitoterapia exerce interferência nos tratamentos farmacológicos tradicionais. Sobre a existência de efeitos negativos causados por fitoterápicos, 4 julgam que não os causam, enquanto 2 reconhecem que esses produtos podem apresentar toxicidade significativa. Esclarece-se que o uso de plantas medicinais e fitoterápicos não é isento de efeitos tóxicos. Os Centros de Informações Toxicológicas (CITs) apontam que a fitoterapia é um recurso terapêutico muito utilizado em automedicação e pode causar intoxicações (CAVALINI et al, 2005). Os entrevistados. em sua maioria $(\mathrm{n}=9)$, têm a percepção de que os medicamentos fitoterápicos são seguros, eficazes e de qualidade. Por outro lado, apenas 5 reconhecem esses produtos como sendo economicamente viáveis para o município.

\section{Uso pessoal de plantas e fitoterápicos}

O uso de plantas medicinais e fitoterápicos pelos próprios coordenadores de unidades foi revelado por 13 dos entrevistados, o que demonstra a aceitaçáo pessoal desses recursos terapêuticos entre os profissionais da saúde.

Entre os que afirmaram fazer uso desses produtos, 3 citaram nomes de produtos industrializados e especificaram as indicaçóes correspondentes, enquanto 8 afirmaram fazer uso de plantas medicinais sob a forma de chás, sendo que apenas a metade destes especificou quais chás são usados. O grupo citou 13 plantas consideradas medicinais: erva-doce, camomila, abacaxi, 
alface, boldo, cáscara-sagrada, copaíba, erva-cidreira, funcho, guaco, laranjeira, marcela e sene. Dessas, duas plantas, a cáscara-sagrada e o guaco, fazem parte do elenco de referências nacional do componente básico da Assistência Farmacêutica (BRASIL, 2010c). Ao analisarmos as enfermidades tratadas com plantas medicinais e fitoterápicos, identificamos maior percentual para o efeito digestivo e calmante (tabela 1).
As plantas medicinais e as indicaçóes citadas foram comparadas à nomenclatura popular das drogas vegetais e a alegaçóes constantes na RDC no 10/2010. Observou-se que as indicaçóes empregadas pelos coordenadores estão de acordo com a legislação, com exceção da planta erva-doce. Essa norma padronizou o uso dos frutos da erva-doce (Pimpinela anisum) para dispepsia, cólicas gastrintestinais e expectorante.

Tabela 1. Indicações para o uso da fitoterapia pelos coordenadores de unidades de saúde. - Porto Alegre, 2010

\begin{tabular}{ll}
\hline Indicações citadas & Frequência \\
\hline Digestivo & $03(18,75 \%)$ \\
\hline Calmante/tranquilizante/ansiedade & $03(18,75 \%)$ \\
\hline Expectorante & $02(12,5 \%)$ \\
\hline Antiinflamatório & $02(12,5 \%)$ \\
\hline Regulação do fluxo intestinal & $01(6,25 \%)$ \\
\hline Triglicerídeos & $01(6,25 \%)$ \\
\hline Antiflatulência & $01(6,25 \%)$ \\
\hline Cólicas & $01(6,25 \%)$ \\
\hline Pirose & $01(6,25 \%)$ \\
\hline Alergia & $01(6,25 \%)$ \\
\hline Total & 16 \\
\hline
\end{tabular}

Fonte: Elaboração Própria.

Uma questão muito importante a considerar é a denominação popular das plantas. Alguns nomes populares consagrados em determinadas regióes correspondem a outros nomes em outras localizaçóes e na literatura. Por exemplo, no sul do país, a planta ervadoce é também chamada regionalmente como 'funcho', porém, essa denominaçáo popular não consta na RDC no 10/2010, literatura de referência da presente pesquisa.

A alface e o abacaxi não são considerados como plantas medicinais na literatura científica. Entretanto, Ritter et al. (2002) referem o uso popular da alface com fins medicinais em algumas pesquisas.

\section{Percepção dos coordenadores sobre a cul- tura de prescrição/indicação de plantas medicinais e fitoterápicos pelos profissio- nais das suas unidades de saúde}

Os coordenadores foram questionados se os profissionais prescritores de suas unidades recomendavam o uso de plantas aos usuários. Nesse sentido, 7 entrevistados responderam haver tal prática, sendo que 5 consideraram que ela ocorre raramente e 2 frequentemente. Das 8 pessoas que assinalaram não, 3 assinalaram que a prática ocorre raramente, contradizendo a resposta negativa sobre a mesma. 
A indicação de fitoterápicos pelos prescritores ocorre em 3 das 15 unidades, não ocorrem em 10 e não houve resposta para 2 unidades. Há a percepção de que os prescritores recomendam mais plantas medicinais do que fitoterápicos. $\mathrm{E}$, quando os coordenadores foram perguntados se eles mesmos já haviam indicado ou prescrito fitoterapia para seus usuários, 10 relataram já ter feito, destes, 8 o fazem eventualmente, 2 habitualmente e 5 afirmaram que nunca o fizeram.

É importante que os gestores de serviços de saúde não fiquem alheios. A existência de práticas complementares necessita ser reconhecida no processo de cuidado. Como anteriormente apontado, as terapias complementares têm como um dos seus objetivos serem utilizadas visando um atendimento integral. Portanto, o fato de que alguns profissionais entrevistados já indicaram plantas medicinais ou fitoterápicos para seus usuários pode ser entendido como uma receptividade positiva ao uso terapêutico de plantas medicinais. A resposta de um dos entrevistados, o qual complementou que apenas indica esses produtos quando solicitado pelo paciente, também demonstra, acima de tudo, a consideração do indivíduo e de suas particularidades; entretanto, há de se investigar se há, nesses casos, compreensão sobre o uso.

\section{Conclusão}

A pesquisa demonstrou a necessidade de capacitação dos trabalhadores do SUS sobre terapias integrativas e complementares. A temática necessita ser explorada em atividades de educação, em algum espaço onde as diferenças conceituais de cada prática possam ser esclarecidas entre os profissionais. Há necessidade, também, do grupo se aproximar da PNPIC e das possibilidades de expansão de suas práticas.
A fitoterapia foi mais indicada para implementação na rede, sendo considerada como uma prática complementar ao tratamento convencional pelos coordenadores. Observou-se a necessidade de informaçâo sobre os seus efeitos indesejáveis e interaçôes. A pesquisa demonstrou que há prática do uso pessoal de plantas medicinais ou fitoterápicos entre profissionais atuantes nos serviços de saúde pública, bem como a indicação desse recurso terapêutico aos usuários. Os coordenadores percebem haver entre os prescritores uma recomendação maior de plantas medicinais em comparação aos fitoterápicos.

Os profissionais e usuários não dispóem de produtos fitoterápicos pela assistência farmacêutica municipal. Sua disponibilização precisa ser debatida entre profissionais da saúde, pesquisadores, gestores e o controle social. É importante que os envolvidos estejam atualizados sobre as oportunidades da inserção de fitoterápicos no âmbito municipal, inclusive das possibilidades de financiamento com recursos financeiros destinados ao Componente da Assistência Farmacêutica Básica.

Conclui-se que os coordenadores de unidades de saúde, assim como acontece entre os usuários, buscam terapias que melhorem seu quadro de saúde, demonstrando, assim, que há uma boa perspectiva de aceitação da implantação da fitoterapia na rede de APS do município em questão.

Reconhece-se a dificuldade de transformação de dados quantitativos obtidos através dos questionários em dados qualitativos que permitam o aprofundamento de cada resposta e do seu contexto real. Sugere-se a continuidade da exploração dessa temática e a aplicação de métodos adicionais de pesquisa na busca de orientações para a efetiva implantação da fitoterapia, entre outras práticas integrativas e complementares, no SUS. 


\section{Referências}

ALVES, M.; PENNA, C. M. M.; BRITO, M. J. M. Perfil dos Gerentes de Unidades Básicas de Saúde. Revista Brasileira de Enfermagem, Brasília, v. 57, n. 4, p. 441-446, 2004.

ALVIM, N. A. T. et al. The use of medicinal plants as a therapeutical resource: from the influences of the professional formation to the ethical and legal implications of its applicability as an extension of nursing care practice. Revista Latino-americana de Enfermagem, Ribeirão Preto, v.14, n. 3, p.316-23, maio/jun. 2006.

BRASIL. Decreto n. 5.813, de 22 de junho de 2006. Aprova a Política Nacional de Plantas Medicinais e Fitoterápicos e dá outras providências. Diário Oficial [da] República Federativa do Brasil, Brasília, DF, 23 jun. 2006a. Seção 1.

Ministério da Saúde. Portaria $n^{\circ} 4.217$, de 28 de dezembro de 2010. Aprova as normas de financiamento e execução do Componente Básico da Assistência Farmacêutica. Brasília: Ministério da Saúde, 2010c.

Ministério da Saúde. Relatório Final. In: Conferência Nacional de Saúde, 8, Brasília, 1986. Brasília: Ministério da Saúde, 1986. 29p.

Ministério da Saúde. Relatório Final. In: Conferência Nacional de Saúde, 10, Brasília, 1996. Brasília: Ministério da Saúde, 1996. $91 \mathrm{p}$.

Ministério da Saúde. Portaria GM no 3.237, de 24 de dezembro de 2007. Assistência Farmacêutica Básica. Brasília, 2007b.

Ministério da Saúde. Portaria Ministerial no 971, de 3 de maio de 2006. Aprova a Política Nacional de Práticas Integrativas e Complementares (PNPIC) no Sistema Único de Saúde. Brasília: Ministério da Saúde, 2006b.

Ministério da Saúde. Secretaria de Ciência, Tecnologia e Insumos Estratégicos. Departamento de Assistência Farmacêutica. A fitoterapia no SUS e o Programa de Pesquisa de Plantas Medicinais da Central de Medicamentos. Brasília: Ministério da Saúde, 2006c.

Ministério da Saúde. Programa Nacional de Plantas Medicinais e Fitoterápicos. Brasília: Ministério da Saúde, 2009a.

CARNEIRO, S. M. O. et al. Da planta ao medicamento: experiência na utilização da fitoterapia na atenção primária à saúde no município de Itapipoca (CE). Divulgação em Saúde para Debate, Rio de Janeiro, n. 30, p.50-55, mar. 2004.

CAVALINI, M. et al. Serviço de informações sobre plantas medicinais e medicamentos fitoterápicos. Extensio: Revista eletrônica de extensão da UFSC, Florianópolis, n. 2, p.1-11, 2005.
CONSELHO FEDERAL DE ENFERMAGEM (COFEN). Resolução no 197, de 19 de março de 1997. Brasília: Conselho Federal de Enfermagem, 1997.

CONSELHO FEDERAL DE FARMÁCIA (CFF). Resolução n 459, de 28 de fevereiro de 2007. Dispõe sobre as atribuições do farmacêutico no âmbito das plantas medicinais e toterápicos e dá outras providências. Brasília: Conselho Federal de Farmácia, 2007.

CONSELHO FEDERAL DE MEDICINA (CFM). Parecer n 04, de 17 de janeiro de 1992. Brasília: Conselho Federal de Medicina, 1992.

CZERMAINSKI, S. B. C. A Política nacional de plantas medicinais e fitoterápicos: um estudo a partir da análise de políticas públicas. 2010. 148f. Dissertação (Mestrado em Ciências Farmacêuticas) Universidade Federal do Rio Grande do Sul, Porto Alegre: UFRGS, 2010.

FONTE, N. N. A transdiciplinaridade como elemento fundamental no setor de plantas medicinais. In: Congresso Mundial de Transdisciplinaridade, 2, 2005, Vitória/Vila Velha. p.1. v. 1.

GRAÇA, C. Treze anos de Fitoterapia em Curitiba. Divulgação em Saúde para Debate, Rio de Janeiro, n.30, p.36-41, mar. 2004.

KULKAMP, I. C. et al. Aceitação de práticas não-convencionais em saúde por estudantes de medicina da Universidade do Sul de Santa Catarina. Revista Brasileira de Educação Médica, Rio de Janeiro, v. 31, n. 3, 229-235, dez. 2007.

KUREBAYASHI, L. F. S.; FREITAS, G. F.; OGUISSO, T. Enfermidades tratadas e tratáveis pela acupuntura segundo percepção de enfermeiras. Revista da Escola de Enfermagem, São Paulo, v. 43, n. 4, p.930-936, dez. 2009.

LUZ NETTO, Memento terapêutico fitoterápico do Hospital das Forças Armadas. Brasília: EGGCF, 1998. 15p.

MATO GROSSO. Governo do Estado . Secretaria Estadual de Saúde. Secretaria do Estado e do Trabalho, Emprego e Cidadania. Fitoplama. DE LA CRUZ, M. G. (org.). O acesso aos fitoterápicos e plantas medicinais e a inclusão social: diagnóstico situacional da cadeia produtiva farmacêutica no estado de Mato Grosso. Mato Grosso: Secretaria Estadual de Saúde, Secretaria do Estado e do Trabalho, Emprego e Cidadania, mar. 2005.

MATOS, F. J. A. Farmácia Vivas. 3.ed. Fortaleza: UFC, 1998. 179p.

MICHILES, E. Diagnóstico situacional dos serviços de fitoterapia no Estado do Rio de Janeiro. Revista Brasileira de Farmacognosia, Curitiba, v. 14. supl. 01, p. 16-19, 2004.

NEVES, L.C. P.; SELLI, L.; JUNGES, R. A integralidade na Terapia Floral e a viabilidade de sua inserção no Sistema Único de Saúde. Mundo saúde, São Paulo, v. 34, n. 1, p. 57-64, jan./mar. 2010. 
OGAVA, S. E. N. et al. Implantação do programa de fitoterapia "Verde Vida" na secretaria de saúde de Maringá (2000-2003). Revista Brasileira de Farmacognosia, Curitiba, v. 13, supl., p. 58-62, 2003.

ORGANIZACIÓN MUNDIAL DE LA SALUD. Pautas para la evaluación de Medicamentos Herbarios. Genebra: OMS, 1991. 5p.

ORGANIZAÇÃO MUNDIAL DE SAÚDE; FUNDO DAS NAÇÕES UNIDAS PARA INFÂNCIA. Conferência Internacional Sobre Cuidados Primários de Saúde: Alma-Ata-URSS, 1978: Relatório final. Brasília: OMS-UNICEF, 1979.

PIRES, A. M.; BORELLA, J. C.; RAYA, L. C. Prática alternativa de saúde na atenção básica da rede SUS de Ribeirão Preto (SP). Divulgação em Saúde para Debate, Rio de Janeiro, n.30, p.56-58, mar. 2004.

PONTES. R. M. F.; MONTEIRO, P. S.; RODRIGUES, M. C. S. O uso da fitoterapia no cuidado de crianças atendidas em um centro de saúde do Distrito Federal. Comunicação em Ciências da Saúde, Brasília, v. 17, n. 2, p.129-139, 2006.

RAMIRES, E. P.; LOURENÇÃO, L. G.; SANTOS, M. R. Gerenciamento em Unidades Básicas de Saúde: conhecendo experiências. Arquivos de Ciência da Saúde, Umuarama, v. 11, n. 4, p. 205-209, out./dez. 2004.

REIS, M.C. et al. Experiência na implantação do programa de fitoterapia do município do Rio de Janeiro. Divulgação em Saúde para Debate, Rio de Janeiro, n. 30, p. 42-49, mar. 2004.

RIO DE JANEIRO. Secretaria Estadual de Saúde. Resolução SES n. 1590, de 12 de fevereiro de 2001. Rio de Janeiro: Secretaria do Estado de Saúde, 12 de fev. 2001.44p.
RIO DE JANEIRO. Decreto n. 23.052, de 16 de abril de 1997. Regulamenta a Lei 2537 de 16 de abril de 1996 que cria o Programa Estadual de Plantas Medicinais. Rio de Janeiro: Diário Oficial do Estado do Rio de Janeiro, 17 abr. 1997. 3p.

RITTER, M.R. et al. Plantas utilizadas como medicinais no município de Ipê, RS. Revista Brasileira de Farmacognosia, Curitiba, v. 12, n.2, p. 51-62, jul./dez. 2002.

SANTOS, L.C. Antônio Moniz de Souza, o 'Homem da Natureza Brasileira': ciência e plantas medicinais no início do século XIX. História, Ciências, Saúde - Manguinhos, Rio de Janeiro, v.15, n.4, p.1025-1038, out./dez. 2008.

SANTOS, F. S. D.. Tradições populares de uso de plantas medicinais na Amazônia. História, Ciências, Saúde - Manguinhos, Rio de Janeiro, v.6, supl., p. 919-939, set. 2000.

SILVA,M.I.G.;GONDIM, A.P.S.;NUNES, F.S. Utilização de fitoterápicos nas unidades básicas de atenção à saúde da família no município de Maracanaú (CE). Revista Brasileira de Farmacognosia, Curitiba, v. 16, n. 4, p. 455-462, out./dez. 2006.

SOUZA, E. F. A. A.; LUZ, M. T. Bases socioculturais das práticas terapêuticas alternativas. História, Ciências, Saúde - Manguinhos, Rio de Janeiro, v. 16, n. 2, p.393-405, abr./jun. 2009.

SPADACIO, C.; BARROS, N. F. Uso de medicinas alternativas e complementares por pacientes com câncer: revisão sistemática. Revista de Saúde Pública, São Paulo, v. 42, n.1, 2008.

TESSER, C. D.; BARROS, N. F. Medicalização social e medicina alternativa e complementar: pluralização terapêutica do Sistema Único de Saúde. Revista de Saúde Pública, São Paulo, v. 42, n. 5, p. 914-920, out. 2008.

Recebido para publicação em Maio/2012

Versão definitiva em Junho/2012

Suporte financeiro: Escola de Saúde Pública do Rio Grande do Sul (ESP/RS) através do Programa de Terceiro Ano de Residência Multiprofissional em Saúde.

Conflito de interesse: inexistente 